\title{
A Pilot Clinical Trial on L-Carnitine Supplementation in Combination with Motivation Training: Effects on Weight Management in Healthy Volunteers
}

\author{
Satoshi Odo ${ }^{1}$, Koji Tanabe ${ }^{1}$, Masamitsu Yamauchi ${ }^{2}$ \\ ${ }^{1}$ Lonza Japan Ltd., Tokyo, Japan; ${ }^{2}$ Department of Home Economics, Registered Dietitians Section, Aichi Gakusen University, Oka- \\ zaki, Japan. \\ Email: Satoshi.odo@lonza.com
}

Received December $4^{\text {th }}, 2012$; revised January $9^{\text {th }}, 2013$; accepted January $16^{\text {th }}, 2013$

\begin{abstract}
A 4-week low dosage (500 mg/day) L-carnitine supplementation in combination with motivation training was carried out in 24 overweight (BMI $25.8-26.6 \mathrm{~kg} / \mathrm{m}^{2}$ ) Japanese males in the course of a double-blind randomized placebo-controlled study. L-carnitine motivated group showed significant body weight loss and a decrement of serum triglyceride level vs. the non-motivated placebo group. Serum adiponectin levels increased in both L-carnitine supplemented groups. The beneficial effects of L-carnitine were amplified by motivation training. For clinical evaluation of supplements, whose efficacy is potentially affected by inter-individual life style variability, supportive motivation training might be advisable for future clinical trials.
\end{abstract}

Keywords: L-Carnitine; Weight Management; Motivation Training; Carnipure ${ }^{\mathrm{TM}}$

\section{Introduction}

Prevalence of obesity among adults in Japan was $3.9 \%$ in 2009 , compared to nearly $34 \%$ for the United States [1]. However, according to national health and nutrition survey in Japan in 2007, prevalence of obesity is increasing in males regardless of age group compared with the statistics of 1986 and 1996. Additionally, among the $40-74$ years old, one in two men and one in five women were strongly suspected to have either metabolic syndrome or prodromal metabolic syndrome [2].

Drugs approved for long-term management of obesity are very few $[3,4]$ they have limited long-term effectiveness, and they have also significant side effects such as hypertension and insomnia for sibutramine [5] and diarrhea and flatulence for orlistat $[6,7]$. On the other hand, long term administration of L-carnitine for fighting of obesity is considered safe [8] in comparison to the previously mentioned medications.

L-carnitine (L-3-hydroxy-4-N-trimethylaminobutyrate) was discovered more than 100 years ago $[9,10]$. More and more new research is conducted on L-carnitine [11, 12]. Among many other functions, L-carnitine is crucial for beta-oxidation, facilitating the transport of longchain fatty acids across the mitochondrial membrane $[13,14]$, as well as moving the waste products such as poorly metabolizable acyl groups from mitochondria to the cytoplasm [15]. Recent research shows that L-carnitine possesses antioxidant capacity and protects plasma components against oxidative damage $[16,17]$.

For long time, L-carnitine has been marketed as a dietary supplement to improve body composition and increase athletic performance [11]. Wutzke and Lorenz have shown that L-carnitine supplementation led to a significant increase in ${ }^{13} \mathrm{C}$-fat oxidation, whereas protein synthesis and breakdown rates remained unchanged in slightly overweight subjects [18]. This was confirmed by a recent study by Wall and coworkers, who found an increase in lipid utilization due to carnitine supplementation in exercising humans [12]. According to Pekala et al., L-carnitine and its esters have been proposed as a treatment for many conditions, including obesity [19].

Most available results arise from studies which employed daily dosages that were higher than those used as dietary supplements; e.g. more than $1000 \mathrm{mg}$ per day. One of the reasons that high dosages were often used in the clinical studies may be the difficulty to obtain clear results of L-carnitine effects due to its indigenous existence in the subjects. Moreover, energy metabolism is easily affected by individual life styles such as daily diet and physical activities, which may bias the effects of Lcarnitine supplementation.

The pilot study presented here was designed to evalu- 
ate effects of low dose L-carnitine supplementation (500 mg per day) on weight loss and other energy metabolism-related parameters. Special focus is directed to the influence of concomitant motivation training on the outcome parameters of the study.

Modern weight loss programs comprise a combination of supplementation with weight-reducing agents and motivation trainings in order to promote changes in life style with regard to eating habits and physical activity that may support the weight-reducing effects [20-23].

\section{Materials and Methods}

\subsection{Study Design}

The trial was designed as a double-blind randomized placebo controlled study. All subjects provided written informed consent before screening. The study protocol was approved by Shiba Palace Clinic Ethics Committee. The study was conducted from September 2009 to December 2009 including subject recruitment and data processing in SOUKEN (Institute of General Health Development Co., Ltd.).

97 male (20 - 60 years [yrs]) Japanese subjects were screened. 24 male subjects (BMI: $25-28 \mathrm{~kg} / \mathrm{m}^{2}$, triglycerides [TG]: 150 - $350 \mathrm{mg} / \mathrm{dL}$, waist circumference: 85 $100 \mathrm{~cm}$, without exercising habit) were allocated to two classes: L-carnitine group (C) (760 mg of L-carnitine tartrate $=500 \mathrm{mg}$ L-carnitine for 4 weeks) and placebo group (NC). Subjects were instructed to ingest 4 capsules/day with water after breakfast. Lonza Japan Ltd. provided Carnipure ${ }^{\mathrm{TM}}$ (L-carnitine L-tartrate), and placebo (cellulose) capsules.

Each class was divided in two groups: motivated (M) and non motivated (NM). Motivation training included a face-to-face $30 \mathrm{~min}$ session with the principal investigator at the baseline visit, where the subjects were informed about L-carnitine and its metabolic functions. Furthermore, subjects learned about the beneficial effect of weight loss on the risk of metabolic diseases. Subjects were encouraged to perform daily physical activities like "taking stairs" instead of "using escalators". Furthermore, it was recommended to reduce calorie intake to 1500 $1800 \mathrm{kcal}$ by taking light meals. Subjects were instructed to use pedometers (step counters) throughout the day to keep record of their physical activity, and to monitor their body weight using bathroom scales provided to them. Both step count and weight were recorded by each subject of the motivated groups on a daily basis. Furthermore, subjects were instructed to report food intake on the 7 days before start of supplementation and on the 7 days prior to the last visit. After 2 weeks, a 15 min follow up interview was held to review and reconfirm the previous instructions. The interviews were conducted in double blinded manner in terms of L-carnitine ingestion.

At baseline and after 4 weeks of supplementation, the following assessments were performed: body fat content was determined by bioelectrical impedance measurement (In Body 3.2, BIOSPACE Co., Ltd., Korea), basal metabolic rate was measured with a gas analyser (Vmax29s, Sensor Medics Co.). In addition blood analyses were performed (see below under Laboratory Assessment).

In the motivated group (M), energy intake, body weight, and amount of physical activity were monitored. Energy intake was calculated from a web diet survey (including a hearing survey with Excel Eiyo-kun Ver. 4. 5 Kenpakusha Ltd., a diet monitoring software). The amount of exercise was surveyed by continuous pedometer measurements.

Subjects were fasting 12 hours prior to each visit. No beverages or food other than mineral water were allowed until blood was drawn on the measurement day. Subjects were prohibited to take any kinds of dietary supplements or functional foods which claim to modify fat or energy metabolism throughout the study. Subjects were asked to avoid irregular activities (lack of sleep, excessive eating or drinking, etc.).

After 2 weeks and after 4 weeks of supplementation, 15 minutes interviews were held to assess subject compliance and monitor potential adverse events.

This study was implemented in adherence with the guidelines of the Declaration of Helsinki.

\subsection{Laboratory Assessment}

The following parameters were measured in the whole blood: white blood cell count, red blood cell count, haemoglobin, haematokrit, mean corpuscular volume (MCV), mean corpuscular haemoglobin $(\mathrm{MCH})$, mean corpuscular haemoglobin concentration and the platelet count.

In the serum the following was measured: total cholesterol, TG, low density lipoprotein (LDL) cholesterol, high density lipoprotein (HDL) cholesterol, adiponectin, urea nitrogen, creatinine, uric acid, aspartate aminotransferase, alanine aminotransferase, $\gamma$-glutamyltransferase, serum insulin, sodium, potassium and chlorine.

One parameter was measured in the plasma: blood glucose.

One parameter was measured in haemolysate: HbA1c.

All measurements were carried out at the research laboratory of BML, Inc. 5-21-3 Sendagaya, Shibuya-ku, Tokyo 151-0051 Japan.

\subsection{Statistical Analysis}

Subjects were randomized so that the means of neutral fat, BMI, peripheral waist circumference, and age screened were as close as possible between all groups. 
The values of all parameters are presented as the mean \pm standard deviation. 3 subjects were excluded from the statistical evaluation. Any unavailable data for any reason were processed as missed data.

All analyses were carried out using SPSS (IBM) Ver. 16. Effects of supplementation (value after 4 weeks) were tested versus baseline data. Furthermore, effect of L-carnitine supplementation with and without motivation training was tested versus the respective placebo group. Correlated t-test was applied at "baseline" and "after 4 weeks" within each group, and Tukey's test was applied between groups at "baseline" and "after 4 weeks" on all variables.

\section{Results}

\subsection{Baseline Values}

The present study is a randomized, placebo-controlled double blind clinical trial to study the effect of 4 weeks intake of low dose $(500 \mathrm{mg} /$ day) L-carnitine in combination with motivation training on body weight and body composition. Of the 24 subjects who entered into the study, all subjects completed the 4 weeks administration, but only 21 were included in the subsequent evaluation. 3 subjects were excluded from the analysis for violation of one inclusion criterion, as they led extraordinary lifestyles being night workers. The demographic characteristics of the study population at baseline are presented in Table 1. Subjects were randomized so that the means of serum TG levels (216 - $228 \mathrm{mg} / \mathrm{dL})$, BMI (25.8 - 26.6 $\left.\mathrm{kg} / \mathrm{m}^{2}\right)$, peripheral waist circumference $(93.3-99.2 \mathrm{~cm})$, and age (40.2 - $44.4 \mathrm{yr})$ were as close as possible between groups. However, body weight was higher in the L-carnitine-motivated group than in the other 3 groups at baseline (see Table 2).

\subsection{Effect of Motivation Training on Life Style}

Although subjects were motivated to keep daily calorie intake between 1500 and $1800 \mathrm{kcal}$, energy intake of most of the subjects exceeded $2500 \mathrm{kcal} /$ day. Energy

Table 1. Baseline demographic characteristics of the subjects $(n=21)$.

\begin{tabular}{ccccc}
\hline & \multicolumn{2}{c}{ L-carnitine } & \multicolumn{2}{c}{ Placebo } \\
\cline { 2 - 5 } & $\begin{array}{c}\text { Motivated } \\
\text { group }\end{array}$ & $\begin{array}{c}\text { Non-motivated } \\
\text { group }\end{array}$ & $\begin{array}{c}\text { Motivated } \\
\text { group }\end{array}$ & $\begin{array}{c}\text { Non-motivated } \\
\text { group }\end{array}$ \\
\hline $\begin{array}{c}\text { Number of } \\
\text { subjects }\end{array}$ & 5 & 6 & 5 & 5 \\
Age (year) & $44.4 \pm 3.5$ & $43.3 \pm 8.2$ & $40.2 \pm 4.8$ & $43.4 \pm 6.5$ \\
Height (cm) & $175.7 \pm 3.8$ & $168.5 \pm 5.1$ & $169.5 \pm 3.2$ & $169.9 \pm 5.8$ \\
\hline
\end{tabular}

mean \pm SD. intake did not change significantly during the intervenetion and was not different between groups (Table 3). In both motivated groups, average step count increased between baseline $(\mathrm{C} / \mathrm{M}=5934 ; \mathrm{NC} / \mathrm{M}=5199)$ and 4 weeks $(\mathrm{C} / \mathrm{M}=8617 ; \mathrm{NC} / \mathrm{M}=6872)$. However, both increases were not found to be statistically significant.

\subsection{Effect of Intervention on Body Weight and Body Composition}

The L-carnitine motivated group showed a significant decrease in body weight (baseline: $82.0 \pm 2.2$, 4 weeks: $80.9 \pm 1.8 \mathrm{~kg}, \mathrm{p}=0.007$ ) within 4 weeks of intervention, while body weight increased or remained constant in all other groups (see Table 2). The body weight change was significantly different between the L-carnitine motivated group and the placebo non-motivated group $(\mathrm{p}=0.0019$; see Figure 1). As shown in Figure 2, body weight decreased in all subjects in the L-carnitine motivated group, in contrast to all other 3 groups.

BMI decreased significantly in L-carnitine motivated group (from $26.6 \mathrm{~kg} / \mathrm{m}^{2}$ at baseline to $26.2 \mathrm{~kg} / \mathrm{m}^{2}$ after 4 weeks), whereas it remained the same or even slightly increased in other 3 groups (see Table 2).

No significant changes were observed in body fat mass, muscle mass and basal metabolism.

\subsection{Effect of Intervention on Blood Lipids}

The L-carnitine motivated group showed the largest decrease in serum TG levels (baseline $218 \pm 45 \mathrm{mg} / \mathrm{dL}, 4$ weeks $145 \pm 42 \mathrm{mg} / \mathrm{dL}$ ) among all other groups (see Table 4 and Figure 3). TG levels were statistically significantly lower in the L-carnitine motivated group than in the placebo non-motivated group at 4 weeks after intake $(p=0.03)$. Changes in triglyceride levels were significantly different between L-carnitine motivated group as well as L-carnitine non-motivated group and placebo non-motivated group $(p=0.014$ and 0.0223 , respectively, Figure 3). On individual level shown on Figure 4, TG changes were observed with some similarities to those on body weight patterns (Figure 2).

Cholesterol levels did not change significantly, but showed positive trends in the L-carnitine motivated group: Total cholesterol decreased slightly in the L-carnitine motivated group ( $2 \mathrm{mg} / \mathrm{dL})$, in comparison to all other groups which showed mild to moderate increases (up to $+22 \mathrm{mg} / \mathrm{dL}$ in the placebo non-motivated group).

LDL cholesterol remained constant in the L-carnitine motivated group but increased in all other groups. The increase reached statistical significance only in the placebo non-motivated group.

HDL cholesterol did not change significantly in any of the groups. 
Effects on Weight Management in Healthy Volunteers

Table 2. Comparison of body weight and body composition between the 4 groups at baseline and after 4 weeks $(n=21)$.

\begin{tabular}{|c|c|c|c|c|c|}
\hline & & \multicolumn{2}{|c|}{ L-carnitine } & \multicolumn{2}{|c|}{ Placebo } \\
\hline & & Motivated group & Non-motivated group & Motivated group & Non-motivated group \\
\hline Number of subjects & & 5 & 6 & 5 & 5 \\
\hline \multirow{2}{*}{ Body weight (kg) } & Baseline & $82.0 \pm 2.2$ & $73.4 \pm 3.6$ & $75.3 \pm 1.6$ & $76.2 \pm 6.4$ \\
\hline & 4 weeks & $80.9 \pm 1.8^{\mathrm{ab}}$ & $73.6 \pm 3.4$ & $75.3 \pm 1.4$ & $76.9 \pm 6.1$ \\
\hline \multirow{2}{*}{ Body mass index $\left(\mathrm{kg} / \mathrm{m}^{2}\right)$} & Baseline & $26.6 \pm 1.1$ & $25.8 \pm 0.6$ & $26.2 \pm 1.0$ & $26.4 \pm 0.6$ \\
\hline & 4 weeks & $26.2 \pm 1.0^{\mathrm{c}}$ & $25.9 \pm 0.6$ & $26.2 \pm 1.1$ & $26.6 \pm 0.5$ \\
\hline \multirow{2}{*}{ Muscle mass (kg) } & Baseline & $58.7 \pm 3.0$ & $53.6 \pm 4.4$ & $54.3 \pm 3.3$ & $56.5 \pm 4.6$ \\
\hline & 4 weeks & $58.0 \pm 2.7$ & $53.8 \pm 3.7$ & $54.6 \pm 2.5$ & $56.8 \pm 4.5$ \\
\hline \multirow{2}{*}{ Body fat mass(kg) } & Baseline & $20.1 \pm 2.4$ & $16.8 \pm 1.4$ & $17.9 \pm 2.6$ & $16.5 \pm 2.1$ \\
\hline & 4 weeks & $19.7 \pm 2.4$ & $16.6 \pm 1.0$ & $17.6 \pm 2.5$ & $17.0 \pm 1.9$ \\
\hline \multirow{2}{*}{ Basal metabolism (kcal/day) } & Baseline & $1590 \pm 59$ & $1512 \pm 147$ & $1546 \pm 94$ & $1566 \pm 161$ \\
\hline & 4 weeks & $1575 \pm 48$ & $1517 \pm 128$ & $1552 \pm 82$ & $1571 \pm 160$ \\
\hline \multirow{2}{*}{ Waist $(\mathrm{cm})$} & Baseline & $99.2 \pm 3.4$ & $93.3 \pm 3.2$ & $95.5 \pm 4.9$ & $94.7 \pm 6.3$ \\
\hline & 4 weeks & $97.9 \pm 2.3$ & $94.2 \pm 3.5$ & $94.5 \pm 4.4$ & $95.2 \pm 6.6$ \\
\hline
\end{tabular}

mean $\pm \mathrm{SD}$; a: $\mathrm{p}<0.01$, for change from baseline at 4 weeks; $\mathrm{b}: \mathrm{p}<0.05$, for L-carnitine motivated group versus L-carnitine non-motivated group; $\mathrm{p}<0.05$, for change from baseline at 4 weeks. Within each group, correlated t-test was applied at "baseline" and "after"; Tukey's test was applied between every two group at "baseline" and "after 4 weeks".

Table 3. Energy intake and step count in the motivated groups at baseline and after 4 weeks $(n=21)$.

\begin{tabular}{cccc}
\hline & & L-carnitine & Placebo \\
\cline { 3 - 4 } & & Motivated group & Motivated group \\
\hline Number of subjects & 5 & 5 \\
\cline { 2 - 4 } Calories (kcal) & Baseline & $1940 \pm 307$ & $2138 \pm 576$ \\
& 4 weeks & $2079 \pm 215$ & $2125 \pm 413$ \\
& Baseline & $5934 \pm 2367$ & $5199 \pm 2473$ \\
Step count (step) & 4 weeks & $8617 \pm 2883$ & $6872 \pm 1375$ \\
\hline
\end{tabular}

mean $\pm \mathrm{SD}$.

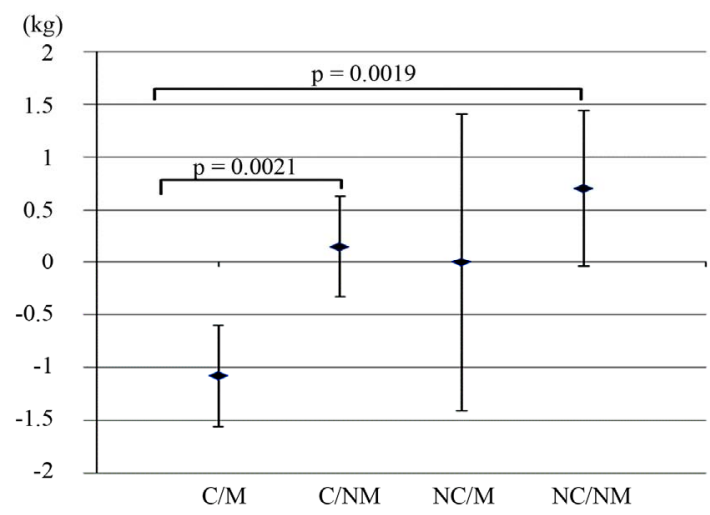

Figure 1. Average body weight change between baseline and 4 weeks. C/M: L-carnitine/Motivated; C/NM: L-carnitine/ Non-motivated; NC/M: Placebo/Motivated; NC/NM: Placebo/Non-motivated. Values are means $\pm \mathrm{SD} ; \mathrm{n}=\mathbf{2 1}$.

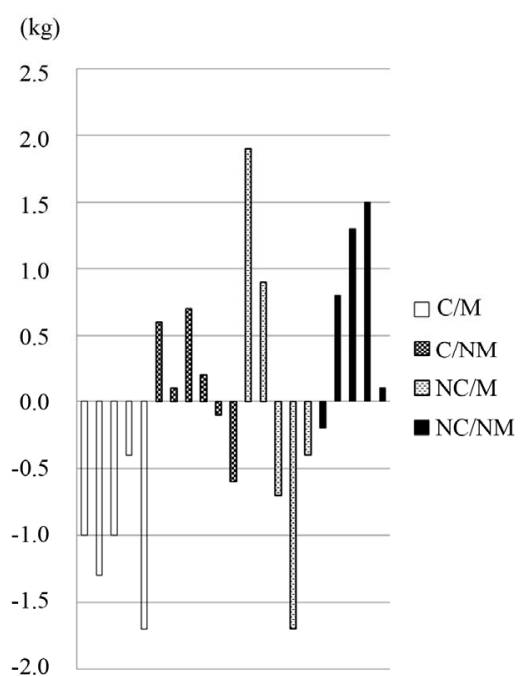

Figure 2. Individual body weight changes between baseline and 4 weeks. C/M: L-carnitine/Motivated; C/NM: L-carnitine/Non-motivated; NC/M: Placebo/Motivated; NC/NM: Placebo/Non-motivated. Values are means $\pm \mathrm{SD} ; \mathrm{n}=\mathbf{2 1}$.

\subsection{Effect of Intervention on Metabolic Parameters}

Adiponectin was significantly higher in the L-carnitine motivated group at 4 weeks after intake compared to baseline (baseline: $7.54 \pm 2.44$, 4 weeks: $8.72 \pm 2.15$ $\mu \mathrm{g} / \mathrm{mL}, \mathrm{p}=0.04$; see Table 5). However, this increase was not statistically significant compared to placebo or L-carnitine non-motivated group. Adiponectin levels 
Effects on Weight Management in Healthy Volunteers

Table 4. Lipid profile between the 4 groups at baseline and after 4 weeks $(n=21)$.

\begin{tabular}{cccccc}
\hline & & \multicolumn{2}{c}{ L-carnitine } & \multicolumn{2}{c}{ Placebo } \\
\cline { 3 - 6 } & & Motivated group & Non-motivated group & Motivated group & Non-motivated group \\
\hline Number of subjects & Baseline & $218 \pm 45$ & 6 & 5 & 5 \\
Triglyceride (mg/dL) & 4 weeks & $145 \pm 42^{\mathrm{a}}$ & $187 \pm 38$ & $183 \pm 51$ & $228 \pm 33$ \\
& Baseline & $215 \pm 31$ & $218 \pm 32$ & $232 \pm 32$ & $209 \pm 28$ \\
Total Cholesterol (mg/dL) & 4 weeks & $213 \pm 36$ & $221 \pm 30$ & $237 \pm 30$ & $231 \pm 36$ \\
& Baseline & $126 \pm 22$ & $128 \pm 18$ & $147 \pm 30$ & $125 \pm 29$ \\
LDL Cholesterol (mg/dL) & 4 weeks & $129 \pm 24$ & $138 \pm 24$ & $154 \pm 25$ & $141 \pm 38^{\mathrm{b}}$ \\
& Baseline & $57.4 \pm 14.3$ & $50.3 \pm 18.8$ & $48.2 \pm 3.6$ & $46.4 \pm 10.9$ \\
HDL Cholesterol (mg/dL) & 4 weeks & $61.0 \pm 14.0$ & $50.2 \pm 12.6$ & $50.8 \pm 6.0$ & $47.2 \pm 10.8$ \\
\hline
\end{tabular}

mean $\pm \mathrm{SD}$; a: $\mathrm{p}<0.05$, for L-carnitine motivated group versus placebo non-motivated group; b: $\mathrm{p}<0.05$, for change from baseline at 4 weeks; Within each group, correlated t-test was applied at "baseline" and "after 4 weeks"; Tukey's test was applied between every two group at "baseline" and "after 4 weeks".

Table 5. Metabolic parameters between the 4 groups at baseline, and after 4 weeks $(n=21)$.

\begin{tabular}{cccccc}
\hline & & \multicolumn{2}{c}{ L-carnitine } & \multicolumn{2}{c}{ Placebo } \\
\cline { 3 - 6 } & & Motivated group & Non-motivated group & Motivated group & Non-motivated group \\
\hline Number of subjects & Baseline & $7.54 \pm 2.44$ & $6.32 \pm 3.74$ & $6.52 \pm 1.75$ & 5 \\
Total adiponectin $(\mu \mathrm{g} / \mathrm{mL})$ & 4 weeks & $8.72 \pm 2.15^{\mathrm{a}}$ & $6.93 \pm 4.60$ & $6.08 \pm 1.48$ & $5.68 \pm 1.26$ \\
& Baseline & $99.2 \pm 3.7$ & $97.2 \pm 4.5$ & $99.8 \pm 9.1$ & $95.24 \pm 0.33$ \\
Blood glucose $(\mathrm{mg} / \mathrm{dL})$ & 4 weeks & $98.6 \pm 2.7$ & $98.7 \pm 11.2$ & $96.2 \pm 4.4$ & $100.0 \pm 4.0$ \\
& Baseline & $5.10 \pm 0.29$ & $5.07 \pm 0.28$ & $4.72 \pm 0.24$ & $4.78 \pm 0.19$ \\
HbAlc $(\%)$ & 4 weeks & $5.12 \pm 0.26$ & $5.10 \pm 0.28$ & $4.72 \pm 0.29$ & $4.82 \pm 0.24$ \\
Insulin $(\mu \mathrm{U} / \mathrm{mL})$ & Baseline & $7.94 \pm 2.16$ & $6.17 \pm 1.80$ & $10.70 \pm 10.20$ & $5.96 \pm 2.44$ \\
& 4 weeks & $8.46 \pm 1.92$ & $13.10 \pm 7.00^{\mathrm{a}, \mathrm{c}}$ & $5.44 \pm 1.58$ & $10.70 \pm 3.70^{\mathrm{b}}$ \\
\hline
\end{tabular}

mean \pm SD; $a: p<0.05$, for change from baseline at 4 weeks; $b$ : $p<0.01$, for change from baseline at 4 weeks; c: $p<0.05$, for L-carnitine non-motivated group versus placebo motivated group; Within each group, correlated t-test was applied at "baseline" and "after 4 weeks"; Tukey's test was applied between every two group at "baseline" and "after 4 weeks".

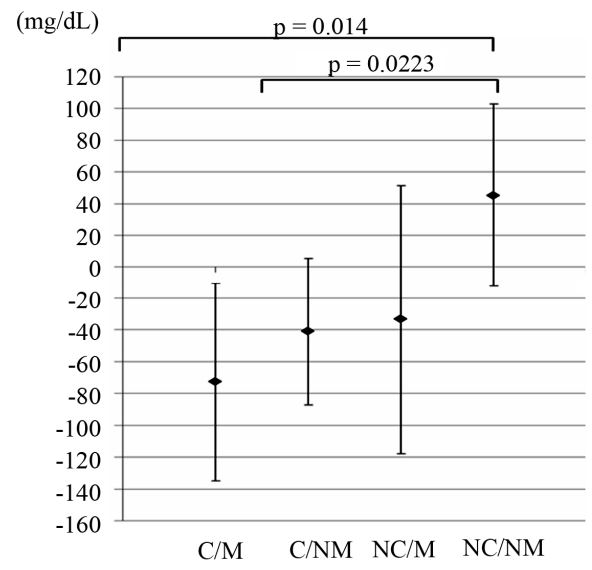

Figure 3. Average changes in serum triglyceride levels between baseline and 4 weeks. C/M: L-carnitine/Motivated; C/ NM: L-carnitine/Non-motivated; NC/M: Placebo/Motivated; NC/NM: Placebo/Non-motivated. Values are means $\pm \mathrm{SD} ; \mathbf{n}=$ 21.

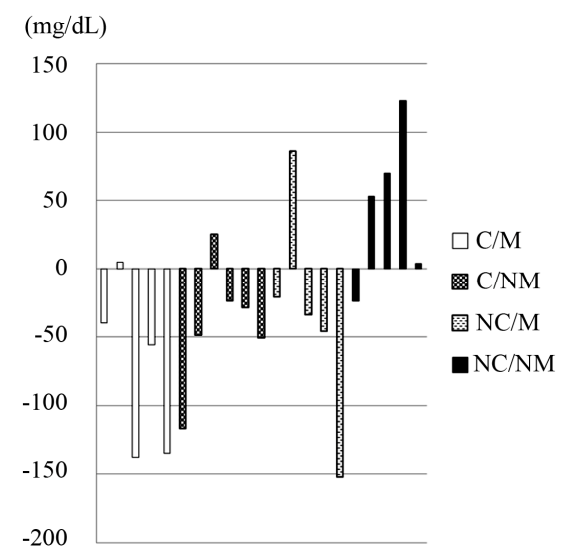

Figure 4. Individual changes in serum triglyceride levels between baseline and 4 weeks. C/M: L-carnitine/Motivated, C/NM: L-carnitine/Non-motivated; NC/M: Placebo/Motivated; NC/NM: Placebo/Non-motivated. Values are means \pm $\mathrm{SD} ; \mathbf{n}=\mathbf{2 1}$. 
showed an increase in all subjects in the L-carnitine motivated group, in contrast to the diversified results of all other 3 groups.

No clinically or statistically significant changes were observed in levels of glucose and HbAlc across the different groups. Insulin levels showed large fluctuations and increased statistically significantly in both carnitine and placebo non-motivated groups compared to baseline $(\mathrm{p}=0.038$ and $\mathrm{p}=0.006$, respectively). Insulin levels were statistically significantly higher in the L-carnitine non-motivated group compared to placebo motivated group at 4 weeks $(\mathrm{p}=0.047)$.

\subsection{Effect of Intervention on Safety Parameters}

Slight changes were observed in white blood cell count, $\mathrm{MCV}, \mathrm{MCH}$, platelet count and plasma urea nitrogen. However, these changes were not clinically relevant and were not confined to a certain treatment group. The results from the complete blood count are presented in Table 6. Markers of renal function and liver function are shown in Table 7. Both L-carnitine and placebo were well tolerated in all subjects.

Table 6. Complete blood count between the 4 groups at baseline and after 4 weeks $(n=21)$.

\begin{tabular}{|c|c|c|c|c|c|}
\hline & & \multicolumn{2}{|c|}{ L-carnitine } & \multicolumn{2}{|c|}{ Placebo } \\
\hline & & Motivated group & Non-motivated group & Motivated group & Non-motivated group \\
\hline Number of subjects & & 5 & 6 & 5 & 5 \\
\hline \multirow{2}{*}{ White blood cells $\left(\times 10^{0} / \mu \mathrm{L}\right)$} & Baseline & $7458 \pm 2261$ & $6243 \pm 1743$ & $4784 \pm 1020$ & $5536 \pm 1422$ \\
\hline & 4 weeks & $5770 \pm 1069$ & $7027 \pm 2818$ & $4886 \pm 1280$ & $6032 \pm 1256^{\mathrm{a}}$ \\
\hline \multirow{2}{*}{ Red blood cells $\left(\times 10^{4} / \mu \mathrm{L}\right)$} & Baseline & $498 \pm 18$ & $519 \pm 19$ & $513 \pm 19$ & $486 \pm 20$ \\
\hline & 4 weeks & $509 \pm 25$ & $522 \pm 31$ & $512 \pm 30$ & $508 \pm 14$ \\
\hline \multirow{2}{*}{ Hemoglobin $(\mathrm{g} / \mathrm{dL})$} & Baseline & $15.4 \pm 0.8$ & $15.4 \pm 0.7$ & $15.7 \pm 0.4$ & $14.8 \pm 0.8$ \\
\hline & 4 weeks & $15.7 \pm 0.9$ & $15.5 \pm 0.9$ & $15.6 \pm 1.1$ & $15.7 \pm 0.6$ \\
\hline \multirow{2}{*}{ Hematocrit (\%) } & Baseline & $45.6 \pm 2.3$ & $47.2 \pm 2.2$ & $46.8 \pm 2.4$ & $44.4 \pm 1.6$ \\
\hline & 4 weeks & $46.1 \pm 2.2$ & $46.4 \pm 2.3$ & $46.2 \pm 2.2$ & $45.9 \pm 1.0$ \\
\hline Platelet count $\left(\times 10^{4} / \mu \mathrm{L}\right)$ & Baseline & $23.0 \pm 4.8$ & $24.6 \pm 3.9$ & $21.7 \pm 4.9$ & $25.2 \pm 7.4$ \\
\hline
\end{tabular}

mean $\pm \mathrm{SD}$; a: $\mathrm{p}<0.05$, for change from baseline at 4 weeks; $\mathrm{b}$ : $\mathrm{p}<0.05$, for change from baseline at 4 weeks; Within each group, correlated t-test was applied at "baseline" and "after 4 weeks"; Tukey's test was applied between every two group at "baseline"and "after 4 weeks".

Table 7. Markers of liver and renal functions between the 4 groups at baseline and after 4 weeks $(\mathrm{n}=\mathbf{2 1})$.

\begin{tabular}{|c|c|c|c|c|c|}
\hline & & \multicolumn{2}{|c|}{ L-carnitine } & \multicolumn{2}{|c|}{ Placebo } \\
\hline & & Motivated group & Non-motivated group & Motivated group & Non-motivated group \\
\hline Number of Subjects & & 5 & 6 & 5 & 5 \\
\hline \multirow{2}{*}{ Creatinine $(\mathrm{mg} / \mathrm{dL})$} & Baseline & $0.82 \pm 0.13$ & $0.79 \pm 0.04$ & $0.84 \pm 0.10$ & $0.83 \pm 0.07$ \\
\hline & 4 weeks & $0.81 \pm 0.16$ & $0.79 \pm 0.06$ & $0.79 \pm 0.10$ & $0.79 \pm 0.03$ \\
\hline \multirow{2}{*}{ Uric Acid (mg/dL) } & Baseline & $6.78 \pm 0.78$ & $6.35 \pm 0.65$ & $6.56 \pm 0.88$ & $6.92 \pm 0.49$ \\
\hline & 4 weeks & $6.78 \pm 1.65$ & $6.33 \pm 1.16$ & $6.42 \pm 0.70$ & $6.88 \pm 1.01$ \\
\hline \multirow{2}{*}{ Urea Nitrogen $(\mathrm{mg} / \mathrm{dL})$} & Baseline & $12.1 \pm 2.4$ & $13.1 \pm 2.3$ & $13.7 \pm 3.7$ & $13.0 \pm 4.2$ \\
\hline & 4 weeks & $16.2 \pm 4.9$ & $13.3 \pm 2.3$ & $13.5 \pm 1.0$ & $15.0 \pm 3.9^{\mathrm{a}}$ \\
\hline \multirow{2}{*}{ AST (U/L) } & Baseline & $22.4 \pm 4.2$ & $26.5 \pm 4.7$ & $22.4 \pm 5.4$ & $23.2 \pm 3.7$ \\
\hline & 4 weeks & $22.2 \pm 4.0$ & $27.2 \pm 8.8$ & $20.2 \pm 4.8$ & $25.4 \pm 6.4$ \\
\hline \multirow{2}{*}{ ALT (U/L) } & Baseline & $28.2 \pm 9.7$ & $39.3 \pm 16.3$ & $20.6 \pm 6.3$ & $23.6 \pm 8.4$ \\
\hline & 4 weeks & $27.4 \pm 7.8$ & $42.3 \pm 21.8$ & $18.2 \pm 6.3$ & $32.0 \pm 17.6$ \\
\hline \multirow{2}{*}{$\gamma$-GT (U/L) } & Baseline & $51.4 \pm 20.5$ & $46.2 \pm 24.7$ & $32.6 \pm 9.7$ & $39.2 \pm 12.1$ \\
\hline & 4 weeks & $49.6 \pm 31.5$ & $47.2 \pm 23.2$ & $31.4 \pm 10.2$ & $58.0 \pm 38.5$ \\
\hline
\end{tabular}

mean $\pm \mathrm{SD}$; a: $\mathrm{p}<0.05$, for change from baseline at 4 weeks; Within each group, correlated t-test was applied at "baseline" and "after 4 weeks"; Tukey's test was applied between every two group at "baseline" and "after 4 weeks". 


\section{Discussion}

The primary purpose of this study was to examine the effect of supplementation with $500 \mathrm{mg}$ of L-carnitine per day for 4 weeks on weight management and energy metabolism related parameters in healthy human subjects with and without supportive motivation training. Although several previous studies have examined Lcarnitine supplementation, this is the first study to report in depth on the effect of low dosage L-carnitine ingestion in combination with motivation training on body weight.

The L-carnitine motivated group showed significant body weight loss of $-1.10 \mathrm{~kg}$ compared to an increase in weight of $+0.7 \mathrm{~kg}$ in the placebo non-motivated group $(\mathrm{p}=$ 0.0019; Figure 1). However, no statistically significant effect of L-carnitine supplementation was observed in the non-motivated group. This is in line with previous studies. Villani et al. found no significant changes in body weight in moderately obese women after supplementation with $4 \mathrm{~g}$ of L-carnitine over 8 weeks [24]. Supplementation with $2 \mathrm{~g}$ of L-carnitine for 3 months in slightly overweight diabetic patients did not affect BMI [25].

It should be noticed that the described study was performed during late fall and winter between September and December 2009. Ma et al. showed that caloric intake, physical activity and subsequently body weight vary seasonally, with mean body weight peaking in winter [26]. This might have also factored into the increase of body weight in the placebo non-motivated group. Despite this seasonal effect on body weight, subjects in the Lcarnitine motivated group in our study showed significant weight loss.

Motivation training comprised information about the beneficial effects of weight loss on the risk of metabolic diseases in face-to-face meetings with the medical instructor as well as instructions to monitor physical activity levels and dietary intake. Participation in the motivation training led to a significant increase of physical activity: In both the placebo motivated and the L-carnitine motivated group, the number of steps increased by +1673 steps and +2683 steps, respectively. Energy intake was not significantly affected by motivation training of the subjects. The administration of L-carnitine in combination with enhanced physical exercise may have led to the observed reduction of body weight by increasing the rate of fat oxidation. Previous studies on both normal and overweight subjects have shown that supplementation with L-carnitine increases fat oxidation [18,27]. Therefore, a reduction of body fat mass may be expected in the L-carnitine motivated group. Although a slight decrease of body fat mass was observed, this change was not statistically significant. However, the short duration of the study may have prevented a significant effect.

Both the L-carnitine motivated group and the Lcarnitine non-motivated group showed a significant drop in serum TG levels compared to the placebo non-motivated group (Figure 3). Figure 5 demonstrates the beneficial effect of a combination of L-carnitine supplementation and motivation on serum TG, decreasing their level below $150 \mathrm{mg} / \mathrm{dL}$ in 3 out of 5 subjects eliminating, hence, a common risk factor for metabolic syndrome [28].

The beneficial effect of L-carnitine on plasma lipids has been documented in several studies [29]. Malaguarnera et al. showed that supplementation with $2 \mathrm{~g}$ of L-carnitine for 3 months significantly reduced TG from $3.31 \pm 0.35$ to $2.30 \pm 0.31 \mathrm{mmol} / \mathrm{L}$ in diabetic patients, which is equivalent to a drop about $-88.4 \mathrm{mg} / \mathrm{dL}$. This is in the same range as the TG change observed in our study.

Malaguarnera et al. also observed positive effects of L-carnitine supplementation on total cholesterol, LDL and HDL cholesterol levels [25]. This has not been demonstrated in our study, where cholesterol values did not change significantly. This may be due to the smaller number of the participating subjects, the lower utilized dose of L-carnitine, or the shorter duration of the trial (4 weeks). Also, in our study, no diet restriction was applied, in contrast to the low-fat and high-fiber diet in the comparative study.

In our study, adiponectin levels were increased in the L-carnitine motivated group at the end of the treatment period compared to baseline levels $(+1.18 \mu \mathrm{g} / \mathrm{ml} ; \mathrm{p}=$ 0.04). A similar trend was observed in the L-carnitine non-motivated group $(+0.61 \mu \mathrm{g} / \mathrm{ml})$, however, this change was not statistically significant. Adiponectin is a peptide hormone produced in the adipocytes, and its levels are inversely related to the fat mass of the body[30]. Administration of adiponectin has been associated

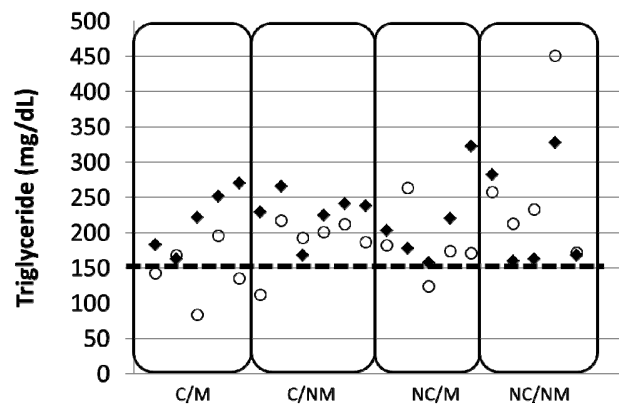

Figure 5. Individual serum triglyceride level at $0 \mathrm{w}(\diamond)$ and $4 \mathrm{w}(\mathrm{O})$. Dotted line (150 $\mathrm{mg} / \mathrm{dL}$ ) shows the critical level for triglycerides as critical risk factor of metabolic syndrome, indicating an amelioration of the risk factor in the L-carnitine motivated group. 
with reduction of plasma glucose levels and increase of insulin sensitivity. Therefore, an increase of adiponectin levels is regarded as beneficial with respect to risk factors of metabolic syndrome [30].

Several studies evaluating changes in adiponectin levels which occurred during weight reduction resulting from various treatments did not have consistent results $[30,31]$. Weight loss per se seems not to have an effect on adiponectin levels. In a study by Silva, de Almeida, and Feoli, weight loss achieved with a low-calorie diet plus exercise increased adiponectin levels in the range of $18 \%-48 \%$ [32]. An increase in adiponectin levels occurred also in a study on the effects of acetyl-L-carnitine (a naturally occurring carnitine derivate, which is deacetylated to L-carnitine after ingestion) in subjects at increased cardiovascular risk [33]. Supplementation with 2 $\mathrm{g} / \mathrm{d}$ acetyl-L-carnitine for 24 weeks (equivalent to $1.6 \mathrm{~g}$ L-carnitine) induced an increase of adiponectin by $27 \%$ compared to an increase about $15 \%$ observed in the Lcarnitine motivated group in our study.

Elevated adiponectin serum levels could be explained by the hypothesis that an improved oxidation of free fatty acids might be associated with a secondary increase in adiponectin expression [34].

Insulin levels showed large variations in our study. The observed significant increases cannot be attributed to either L-carnitine supplementation or motivation training and may have been influenced by other non-controlled parameters.

Our study demonstrates that the efficacy of a weight loss program may be strongly influenced by motivation training. For all parameters that showed significant changes during the treatment (body weight, triglycerides, and adiponectin levels), stronger effects were observed in the L-carntine motivated group than in the L-carnitine nonmotivated group. This is supported by the study of Unick et al. who investigated the influence of a telephone motivation program on the achievement of weight loss in overweight and obese women [35]. It turned out that the number of telephone calls was a strong predictor of weight loss success.

Several countries have recently started health programs to achieve lifestyle changes in order to support weight loss and prevent metabolic diseases [36,37]. Our results substantiate the beneficial effect of motivation training on the efficacy of weight loss programs. Also for the clinical examination of the efficacy of dietary supplements, which may be affected by inter-individual life style variability, the implementation of motivation training may be advisable to obtain more pronounced effects of the intervention.

Supplementation of L-carnitine is considered safe for doses up to $15 \mathrm{~g} / \mathrm{d}$ in healthy men [38]. Therefore, markers of hepatic and renal function as well as blood hematology in Japanese men after daily ingestion of a dose of L-carnitine of $500 \mathrm{mg} / \mathrm{d}$ for 4 weeks showed no abnormalities as expected.

There were some limitations to this pilot study. The main limitation concerns the small number of subjects. This may be a crucial limitation that weakens the significance of the results, although the observed changes do signify a beneficial effect of L-carnitine supplementation in combination with motivation training. We tried to equally allocate subjects to each group, but 3 subjects were excluded for violation of the inclusion criteria. Furthermore, the relatively short duration of this clinical trial (4 weeks) and the utilized low dose of L-carnitine may explain the lack of significance in the achieved changes in many of the assessed parameters. L-carnitine seems to induce a slow but consistent increase of muscle carnitine stores. A recent study by Wall et al. has shown, that an increase in muscle carnitine is observed after 12 weeks of supplementation, which reaches statistical significance only after 24 weeks [12]. Therefore, it can be assumed that the effect of L-carnitine on body weight and serum lipids will be more pronounced in a study of a longer duration.

Low dosage L-carnitine combined with motivation may have a beneficial effect on several metabolic syndrome risk parameters, including TG and adiponectin, which could offer a safe, low cost, and easily applicable strategy targeting weight loss in overweight humans.

\section{REFERENCES}

[1] Organization for Economic Co-operation and Development, "Health at a Glance," OECD Publishing, Paris, 2011 .

[2] Ministry of Health, Labour and Welfare, "Outline for the Results of the National Health and Nutrition Survey Japan," Ministry of Health, Labour and Welfare, Tokoy, 2007.

[3] U. Pagotto, D. Vanuzzo, V. Vicennati and R. Pasquali, "Pharmacological Therapy of Obesity," Giornale Italiano di Cardiologia (Rome), Vol. 9, No. 4, 2008, pp. 83S-93S.

[4] D. S. Weigle, "Pharmacological Therapy of Obesity: Past, Present, and Future," The Journal of Clinical Endocrinology \& Metabolism, Vol. 88, No. 6, 2003, pp. 2462-2469. doi:10.1210/jc.2003-030151

[5] W. P. James, I. D. Caterson, W. Coutinho, N. Finer, L. F. Van Gaal, A. P. Maggioni, C. Torp-Pedersen, A. M. Sharma, G. M. Shepherd, R. A. Rode and C. L. Renz, "Effect of Sibutramine on Cardiovascular Outcomes in Overweight and Obese Subjects," The New England Journal of Medicine, Vol. 363, No. 10, 2010, pp. 905-917.

[6] A. J. Scheen, "Cardiovascular Risk-Benefit Profile of Sibutramine," American Journal of Cardiovascular Drugs, Vol. 10, No. 5, 2010, pp. 321-334. 
doi:10.2165/11584800-000000000-00000

[7] M. Li and B. M. Cheung, "Pharmacotherapy for Obesity," British Journal of Clinical Pharmacology, Vol. 68, No. 6, 2009, pp. 804-810.

[8] M. R. Rubin, J. S. Volek, A. L. Gomez, N. A. Ratamess, D. N. French, M. J. Sharman and W. J. Kraemer, "Safety Measures of L-Carnitine L-Tartrate Supplementation in Healthy Men," The Journal of Strength \& Conditioning Research, Vol. 15, No. 4, 2001, pp. 486-490.

[9] V. S. Gulewitsch and G. R. Krimberg, "Information about Muscle Extracts," Hoppe-Seyler's Journal of Physiological Chemistry, Vol. 45, No. 3-4, 1905, pp. 326-330.

[10] E. Strack, P. Wordehoff, E. Neubaur and H. Geissendörfer, "Information about Muscle Contents of Choline, Acetylcholine and Carnitine," Hoppe-Seyler's Journal of Physiological Chemistry, Vol. 233, No. 5-6, 1935, pp. 189-203.

[11] K. Sahlin, "Boosting Fat Burning with Carnitine: An Old Friend Comes out from the Shadow," The Journal of Physiology, Vol. 589, 2011, pp. 1509-1510. doi:10.1113/jphysiol.2011.205815

[12] B. T. Wall, F. B. Stephens, D. Constantin-Teodosiu, K. Marimuthu, I. A. Macdonald and P. L. Greenhaff, "Chronic Oral Ingestion of L-Carnitine and Carbohydrate Increases Muscle Carnitine Content and Alters Muscle Fuel Metabolism during Exercise in Humans," The Journal of Physiology, Vol. 589 Pt 4, 2011, pp. 963-973.

[13] F. B. Stephens, D. Constantin-Teodosiu and P. L. Greenhaff, "New Insights Concerning the Role of Carnitine in the Regulation of Fuel Metabolism in Skeletal Muscle," The Journal of Physiology, Vol. 581, 2007, pp. 431-444.

[14] D. W. Foster, "The Role of the Carnitine System in Human Metabolism," Annals of the New York Academy of Sciences, Vol. 1033, No. 1, 2004, pp. 1-16. doi:10.1196/annals.1320.001

[15] S. Abdel-Aleem, M. Sayed-Ahmed, M. A. Nada, S. C. Hendrickson, J. St. Louis and J. E. Lowe, "Stimulation of Non-Oxidative Glucose Utilization by L-Carnitine in Isolated Myocytes," Journal of Molecular and Cellular Cardiology, Vol. 27, No. 11, 1995, pp. 2465-2472. doi:10.1006/jmcc.1995.0234

[16] D. Monti, L. Troiano, F. Tropea, E. Grassilli, A. Cossarizza, D. Barozzi, M. C. Pelloni, M. G. Tamassia, G. Bellomo and C. Franceschi, "Apoptosis-Programmed Cell Death: A Role in the Aging Process?" The American Journal of Clinical Nutrition, Vol. 55, No. 6, 1992, pp. 1208S$1214 \mathrm{~S}$.

[17] J. Kolodziejczyk, J. Saluk-Juszczak and B. Wachowicz, "L-Carnitine Protects Plasma Components against Oxidative Alterations," Nutrition, Vol. 27, No. 6, 2011, pp. 693-699. doi:10.1016/j.nut.2010.06.009

[18] K. D. Wutzke and H. Lorenz, "The Effect of L-Carnitine on Fat Oxidation, Protein Turnover, and Body Composition in Slightly Overweight Subjects," Metabolism, Vol. 53, No. 8, 2004, pp. 1002-1006. doi:10.1016/j.metabol.2004.03.007

[19] J. Pekala, B. Patkowska-Sokola, R. Bodkowski, D. Jamroz, P. Nowakowski, S. Lochynski and T. Librowski,
"L-Carnitine-Metabolic Functions and Meaning in $\mathrm{Hu}-$ mans Life," Current Drug Metabolism, Vol. 12, No. 7, 2011, pp. 667-678. doi:10.2174/138920011796504536

[20] J. W. Anderson, L. R. Reynolds, H. M. Bush, J. L. Rinsky and C. Washnock, "Effect of a Behavioral/Nutritional Intervention Program on Weight Loss in Obese Adults: A Randomized Controlled Trial," Postgraduate Medical, Vol. 123, No. 5, 2011, pp. 205-213.

[21] L. F. DeFina, L. G. Marcoux, S. M. Devers, J. P. Cleaver, and B. L. Willis, "Effects of Omega-3 Supplementation in Combination with Diet and Exercise on Weight Loss and Body Composition," The American Journal of Clinical Nutrition, Vol. 93, No. 2, 2011, pp. 455-462. doi:10.3945/ajen.110.002741

[22] D. Laddu, C. Dow, M. Hingle, C. Thomson and S. Going, "A Review of Evidence-Based Strategies to Treat Obesity in Adults," Nutrition in Clinical Practice, Vol. 26, No. 5, 2011, pp. 512-525. doi:10.1177/0884533611418335

[23] C. N. Poole, M. D. Roberts, V. J. Dalbo, P. S. Tucker, K. L. Sunderland, N. D. DeBolt, B. W. Billbe and C. M. Kerksick, "The Combined Effects of Exercise and Ingestion of a Meal Replacement in Conjunction with a Weight Loss Supplement on Body Composition and Fitness Parameters in College-Aged Men and Women," The Journal of Strength \& Conditioning Research, Vol. 25, No. 1, 2011, pp. 51-60. doi:10.1519/JSC.0b013e3181fee4aa

[24] R. G. Villani, J. Gannon, M. Self and P. A. Rich, "L-Carnitine Supplementation Combined with Aerobic Training Does Not Promote Weight Loss in Moderately Obese Women," International Journal of Sport Nutrition and Exercise Metabolism, Vol. 10, No. 2, 2000, pp. 199-207.

[25] M. Malaguarnera, M. Vacante, T. Avitabile, L. Cammalleri and M. Motta, "L-Carnitine Supplementation Reduces Oxidized LDL Cholesterol in Patients with Diabetes," The American Journal of Clinical Nutrition, Vol. 89, No. 1, 2009, pp. 71-76. doi:10.3945/ajen.2008.26251

[26] Y. Ma, B. C. Olendzki, W. Li, A. R. Hafner, D. Chiriboga, J. R. Hebert, M. Campbell, M. Sarnie and I. S. Ockene, "Seasonal Variation in Food Intake, Physical Activity, and Body Weight in a Predominantly Overweight Population," European Journal of Clinical Nutrition, Vol. 60, No. 4, 2006, pp. 519-528. doi:10.1038/sj.ejen.1602346

[27] D. M. Muller, H. Seim, W. Kiess, H. Loster and T. Richter, "Effects of Oral L-Carnitine Supplementation on in Vivo Long-Chain Fatty Acid Oxidation in Healthy Adults," Metabolism, Vol. 51, No. 11, 2002, pp. 1389-1391.

[28] S. M. Grundy, J. I. Cleeman, S. R. Daniels, K. A. Donato, R. H. Eckel, B. A. Franklin, D. J. Gordon, R. M. Krauss, P. J. Savage, S. C. Smith, J. A. Spertus and F. Costa, "Diagnosis and Management of the Metabolic Syndrome," Circulation, Vol. 112, No. 17, 2005, pp. e285-e290.

[29] C. R. Sirtori, L. Calabresi, S. Ferrara, F. Pazzucconi, A. Bondioli, D. Baldassarre, A. Birreci and A. Koverech, "LCarnitine Reduces Plasma Lipoprotein(a) Levels in Patients with Hyper Lp(a)," Nutrition, Metabolism \& Cardiovascular Diseases, Vol. 10, No. 5, 2000, pp. 247-251.

[30] N. Stefan and M. Stumvoll, “Adiponectin-Its Role in 
Metabolism and Beyond," Hormone and Metabolic Research, Vol. 34, No. 9, 2002, pp. 469-474. doi:10.1055/s-2002-34785

[31] M. R. Manigrasso, P. Ferroni, F. Santilli, T. Taraborelli, M. T. Guagnano, N. Michetti and G. Davi, "Association between Circulating Adiponectin and Interleukin-10 Levels in Android Obesity: Effects of Weight Loss," The Journal of Clinical Endocrinology \& Metabolism, Vol. 90, No. 10, 2005, pp. 5876-5879. doi:10.1210/jc.2005-0281

[32] F. M. Silva, J. C. de Almeida and A. M. Feoli, "Effect of Diet on Adiponectin Levels in Blood," Nutrition Reviews, Vol. 69, No. 10, 2011, pp. 599-612. doi:10.1111/j.1753-4887.2011.00414.x

[33] P. Ruggenenti, D. Cattaneo, G. Loriga, F. Ledda, N. Motterlini, G. Gherardi, S. Orisio and G. Remuzzi, "Ameliorating Hypertension and Insulin Resistance in Subjects at Increased Cardiovascular Risk: Effects of Acetyl-L-Carnitine Therapy," Hypertension, Vol. 54, No. 3, 2009, pp. 567-574. doi:10.1161/HYPERTENSIONAHA.109.132522

[34] J. A. Paniagua, A. G. de la Sacristana, I. Romero, A. Vidal-Puig, J. M. Latre, E. Sanchez, P. Perez-Martinez, J. Lopez-Miranda and F. Perez-Jimenez, "Monounsaturated Fat-Rich Diet Prevents Central Body Fat Distribution and
Decreases Postprandial Adiponectin Expression Induced by a Carbohydrate-Rich Diet in Insulin-Resistant Subjects," Diabetes Care, Vol. 30, No. 7, 2007, pp. 1717-1723. doi: $10.2337 / \mathrm{dc} 06-2220$

[35] J. L. Unick, J. M. Jakicic and B. H. Marcus, "Contribution of Behavior Intervention Components to 24-Month Weight Loss," Medicine \& Science in Sports \& Exercise, Vol. 4, No. 4, 2010, pp. 745-753. doi:10.1249/MSS.0b013e3181bd1a57

[36] G. Boehm, N. Bracharz and R. Schoberberger, "Evaluation of the Sustainability of the Public Health Program Slim without Diet (Schlank Ohne Diat)," Wiener Klinische Wochenschrift, Vol. 123, No. 13-14, 2011, pp. 415-421.

[37] C. M. Swanson, S. Bersoux, M. H. Larson, R. T. AponteFurlow, S. S. Flatten, C. L. Olsen, C. Larosa, P. M. Verona, K. A. Jameson and C. B. Cook, "An OutpatientBased Clinical Program for Diabetes Prevention: An Update," Endocrine Practice, Vol. 18, No. 2, 2012, pp. 200208.

[38] J. F. Borzelleca, I. C. Munro and J. A. Thomas, "Evaluation of L-Carnitine for GRAS Status," Report for Lonza Inc., Switzerland, 2011. 